\title{
BCL-2 and MYC gain/amplification is correlated with central nervous system involvement in diffuse large $B$ cell lymphoma at leukemic phase
}

Dehui Zou ${ }^{1 \dagger}$, Shuhua Yi ${ }^{1 \dagger}$, Rui Cui ${ }^{1,2}$, Wei Liu', Chengwen Li ${ }^{1}$, Shizhen Zhong ${ }^{1}$, Zhen Yu' ${ }^{1}$, Zengjun Li ${ }^{1}$, Rui Lv ${ }^{1}$, Kun $\mathrm{Ru}^{1}$, Huijun Wang ${ }^{1}$, Gang $A n^{1}$, Yan $X u^{1}$ and Lugui Qiu ${ }^{1 *}$

\begin{abstract}
Background: Diffuse large B-cell lymphoma (DLBCL) of leukemic phase is a rare clinical manifestation, but is highly prevalent with central nervous system involvement (CNSI). Little is known about this rare clinical observation.

Methods: We reviewed the clinical characteristics of 40 DLBCL patients with leukemic phase identified by flow cytometry and analyzed BCL2 and MYC aberrations by fluorescence in situ hybridization.

Results: The median age of these 40 patients was 46 years (range, 15-75) with 19 men patients. All patients had bone marrow involvement, and fourteen (35.0\%) had CNSI. There were respectively 14 patients (35.0\%) had the BCL2 or MYC gain/amplification and nine of them (22.5\%) simultaneously had both aberrations. Compared to those without CNSI, CNSI was found more commonly in male patients (71.4 vs. $34.6 \%, p=0.046$ ), in those with IPI scores of $4-5(57.1 \%$ vs. $11.5 \%, p=0.001)$, and in those with elevated serum LDH (100 vs. $61.5 \%, p=0.007)$ and both MYC and $\mathrm{BCL} 2$ rearrangement (88.9 vs. 19.4\%; $p=0.000$ ). BCL2 and MYC rearrangements were the sole independent factor correlated with CNSI.
\end{abstract}

Conclusion: It is possible that both BCL2 and MYC gene aberrations may contribute to the high incidence of CNSI observed in leukemic phase of patients with DLBCL.

Keywords: Diffuse large B cell lymphoma, Leukemic phase, Centre nervous system involvement, BCL2, MYC

\section{Background}

Diffuse large B-cell lymphoma (DLBCL) is the most common lymphoid neoplasm, which account for $30 \%-40 \%$ of non-Hodgkin lymphoma (NHL) [1] in the Western countries and more than $50 \%$ of NHL in China. DLBCL is a heterogeneous group of disorders with variable histological and clinical behavior. Up to $40 \%$ of patients may have extranodal involvement at diagnosis. The common extranodal sites include the gastrointestinal tract, bone, bone marrow, testis, salivary gland, adrenal gland, liver,

\footnotetext{
* Correspondence: qiulg@ihcams.ac.cn

${ }^{\dagger}$ Equal contributors

'State Key Laboratory of Experimental Hematology, Institute of Hematology and Blood Disease Hospital, Chinese Academy of Medical Sciences and Peking Union Medical College, No.288, Nanjing road, Heping district, Tianjin 300020, China

Full list of author information is available at the end of the article
}

kidney, and central nervous system (CNS) [1]. The extent of extranodal involvement can impact the overall prognosis of each patient [2]. For example, patients with CNS involvement, which is observed in up to $2 \%-8 \%$ of patients, have poorer outcomes [2-5]. Tumor cells circulated in the peripheral blood $(\mathrm{PB})$ occur rarely in patients with DLBCL. However, approximately $16 \%$ of patients at leukemic phase have CNS involvement, which is associated with increased mortality [6]. It remains unknown why patients with leukemic phase have a higher incidence of CNS involvement. As the double hit with MYC and BCL2 gene rearrangements contribute to the high aggressive behavior of DLBCL [7], we analyzed the cytogenetic aberrations of patients with DLBCL at leukemic phase. We observed that both BCL-2 and MYC genes gain/ 
amplification were strong independent indicators of CNS involvement in DLBCL patients at leukemic phase.

\section{Methods \\ Patients}

A flow cytometry database was searched to identify patients with NHL and with circulating lymphoma cells between August 2001 and May 2012 at Institute of Hematology and Blood Disease Hospital, Chinese Academy of Medical Sciences and Peking Union Medical College (CAMS \& PUMC). The target antigens for flow cytometry included CD19, CD20, CD22, CD10, CD23, FMC7, kappa, lambda, CD3, CD5, CD45, CD11c, CD103 and sIgM. All antibodies were obtained from BD Biosciences.

Finally, forty patients with de novo DLBCL and with complete medical records were enrolled in this study. $\mathrm{Pa}-$ tients with secondary DLBCL were excluded by the clinical course and medical history. Basic demographics (age, gender), performance status, Ann Arbor stage, presence of extra nodal sites, chromosome karyotype, bone marrow biology and peripheral blood morphology, cerebrospinal fluid (CSF) involvement, hematological parameters, lactate dehydrogenase (LDH) levels, and treatment outcomes were assessed. Histologic specimens were reviewed by hematopathologists, including two of the authors of this study, based on WHO classification [1]. All bone marrow tissues evaluated in this study were performed at the time of initial staging. All patients enrolled gave informed consent in accordance with the Declaration of Helsinki. The study was approved by the Ethics Committee of CAMS \& PUMC.

CNS involvement was defined based on the combination of clinical CNS manifestations, radiological findings, and/or examination of the cerebrospinal fluid (CSF). Cytology study of CSF and CNS imaging by brain magnetic resonance imaging (MRI)/computerized tomography $(\mathrm{CT})$ were performed for patients with a clinical suspicion of CNS involvement. The patterns of CNS involvement were as follows: 3 patients identified as parenchymal involvement, 9 patients with leptomeningeal involvement and 2 patients with combined involvement. Five patients were presented with CNS involvement at diagnosis and others at progression or disease recurrence.

\section{Fluorescence In Situ Hybridization (FISH)}

Interphase FISH analysis was performed on bone marrow samples at diagnosis. The DNA probes used were LSI BCL2 and MYC Dual Color, Break Apart Rearrangement Probe, LSI IGH/BCL2 Dual Color, Dual Fusion Translocation Probe and LSI IGH/MYC/CEP 8 Tri-Color Dual Fusion Probes (purchased from Vysis, USA). Sample preparations and hybridizations were conducted following manufacturer's recommendations. Methods for FISH analysis are described elsewhere $[8,9]$. At least 200 cells with well delineated signals were evaluated. The cut-off for positive values (mean of normal control +3SD), determined from samples of ten cytogenetically normal persons, were $4.5 \%$ for LSI BCL2 and MYC Dual Color, Break Apart Rearrangement Probe and 3.2\% for LSI IGH/BCL2 Dual Color, Dual Fusion Translocation Probe and LSI IGH/MYC/CEP 8 Tri-Color Dual Fusion Probes. Gains were defined as three copies of the gene studied, whereas at least four copies were considered as amplifications [10].

\section{Survival and statistical analysis}

Overall survival (OS) was measured as the interval between the date of treatment and the date of death or last follow-up. Progression-free survival (PFS) was measured as the interval between the date of treatment and the date of death from any cause or disease progression. Fisher's exact test or chi-square test was used to determine statistically significant differences between the clinical characteristics of the two groups. Survival curves were constructed by the Kaplan-Meier method, and prognostic features were evaluated on univariate analysis (log-rank test). The effects of potential prognostic variables on survival were assessed according to the Cox regression method. P values $<0.05$ were considered statistically significant. All calculations were performed using the SPSS statistical software package (Version 13.0).

\section{Results \\ Clinical characteristics}

The clinical characteristics of the 40 patients are presented in Table 1. In this study, there were a large number of younger patients, with a median age of 46 years (range 15-75). The median white blood cell (WBC) was $12.32 \times$ $10^{9} / \mathrm{L}$, while the median percentage of circulating lymphomatous cells was $35.74 \%$ (range, 1-90) as determined by flow cytometry. Twenty-seven patients (67.5\%) had anemia and half of these patients had reduced platelet at diagnosis. All of the 40 patients had bone marrow involvement, and 14 patients had CNS involvement. Other extranode sites involvement included lung, kidney, pancreas, adrenal gland, liver, testis, and bowel.

As there was a high percentage of CNS involvement in these patients at leukemic phase, we compared the clinical characteristics between patients with or without CNS involvement (Table 1). There was a significant male preference, higher elevated LDH percentage and high risk IPI group in patients with CNS involvement. The median age of the patients with CNS involvement was also younger than those without ( 44 vs. 51 years, $p=0.392$ ).

\section{Cytogenetic aberrations}

Using the bone marrow cells, we detected BCL2 and MYC gene arrangements by FISH. There was no $\mathrm{t}(14 ; 18)$ (q32;q21) abnormality detected by BCL2/IGH Dual Fusion Translocation Probe. However, 14 patients had 
Table 1 the comparison of clinical characteristics between patients with or without CNS involvement

\begin{tabular}{|c|c|c|c|}
\hline Characteristics & With CNS involvement & Without CNS involvement & $P$ value \\
\hline & $\mathrm{N}=14$ & $N=26$ & \\
\hline Age, median (range, year) & $44.0(23.0-62.0)$ & $51.0(15-75)$ & .392 \\
\hline Gender, men, n(\%) & $9(34.6 \%)$ & $10(71.4 \%)$ & .046 \\
\hline B symptoms, n (\%) & $10(71.4 \%)$ & $15(57.7 \%)$ & .502 \\
\hline Elevated LDH, n (\%) & 14 (100\%) & $16(61.5 \%)$ & .007 \\
\hline Median WBC×109/I (range) & $17.2(1.2-66.57)$ & $9.58(1.38-40.06)$ & .729 \\
\hline Median Hb, g/l (range) & $95.0(61.0-145.0)$ & $100.5(45.0-141.0)$ & .777 \\
\hline Median PLT, ×109/I (range) & $73.5(20.0-355.0)$ & 113.0(3.0-509.0) & .843 \\
\hline International Prognostic & & & .002 \\
\hline Index, n (\%) & 0 & $9(34.6 \%)$ & \\
\hline 2 & $6(42.9 \%)$ & 14(53.8\%) & \\
\hline 3 & $8(57.1 \%)$ & $3(11.5 \%)$ & \\
\hline $4-5$ & & & .896 \\
\hline Treatment, n (\%) & $4(28.6 \%)$ & $10(38.5 \%)$ & \\
\hline CHOP/CHOP-like & $1(7.1 \%)$ & $3(11.5 \%)$ & \\
\hline R- CHOP/CHOP-like & $4(28.6 \%)$ & $5(19.2 \%)$ & \\
\hline Intensive regimes & $5(35.7 \%)$ & $8(30.8 \%)$ & \\
\hline
\end{tabular}

three or more fusion signal of BCL2 (Fig. 1), which meant that $35 \%$ patients had BCL2 gene gain/amplification. Additionally, gain/amplification of MYC was presented in 14 patients (Fig. 1), with one having concomitant MYC/IGH translocation. Nine patients exhibited both $M Y C$ and BCL2 gene gains/amplifications. As shown in Fig. 1, gain and amplification always concurred in one patient. Some patients display predominant amplifications with minor gains and others reversely. So we did not discriminate gain and amplification in an individual here and put gain and amplification together to analyze and used "abnormality" instead of "gain" or "amplification" in this manuscript.

Eight of the fourteen patients (57.1\%) with BCL2 abnormality had CNS involvement, while 6 of 26 patients (23.1\%) that lacked the BCL2 abnormality had CNS involvement $(p=0.043)$. Eleven of the 14 patients $(78.6 \%)$ with MYC abnormality had CNS involvement, which was significant higher than three of the 26 patients (11.5\%) without MYC abnormality $(p<0.001)$. Among the 9 patients with both MYC and BCL2 abnormality, eight patients had CNS involvement, while only 6 of the other 31 patients with BCL2 or MYC or no abnormality had CNS involvement (88.9 vs. $19.4 \% ; p<0.001$ ).

Other clinical characteristics that were associated with CNS involvement (as shown above) included gender, elevated LDH, and high risk IPI group. We performed a multivariate analysis to determine the relationship between the clinical characteristics and CNS involvement, by integrating gender, elevated LDH, high risk IPI, and concomitant BCL2 and MYC abnormalities. We demonstrated that concomitant BCL2 and MYC abnormalities were the only independent factor that correlated with CNS involvement (relative risk 15.3, 95\% confidence

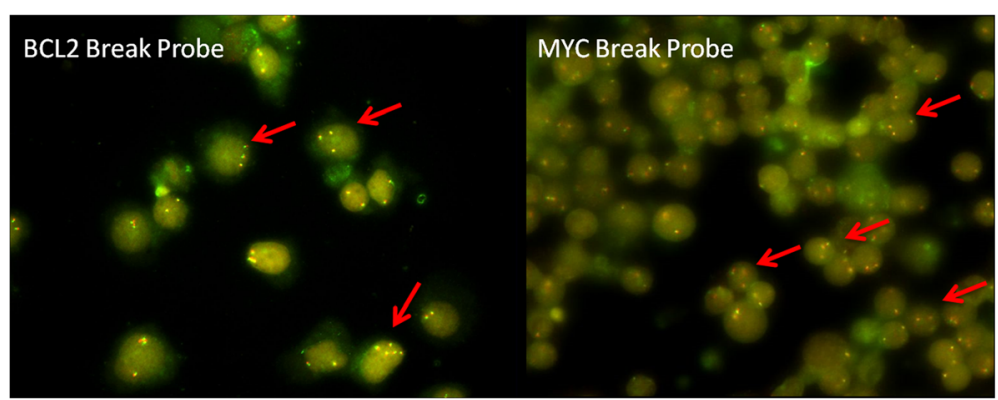

Fig. 1 Genetic aberrations of BCL2 and MYC gene as detected by FISH. The red arrow indicated gene gain or amplification (three or more fusion signals) 
interval, 1.4-171.1, $p=0.027)$. Therefore, there was a strong association between DLBCL patients with concomitant of BCL2 and MYC abnormalities and CNS involvement.

\section{Treatment and survival}

All of the 40 patients included in this study had received at least 2 cycles of chemotherapy, with median 4 cycles (range 2-10). As shown in Table 1, fourteen patients had received $\mathrm{CHOP}$ or $\mathrm{CHOP}$-like regimens, while nine patients had received HyperCVAD/MA alternating chemotherapy or $\mathrm{CHOPE} / \mathrm{EPOCH}$ regimens chemotherapy. Seventeen patients had received rituximab combination chemotherapy, including 4 patients with R-CHOP/ $\mathrm{CHOP}$-like regimens and other with $\mathrm{R}$-intensive regimens. Three patients received R-HyperCVAD/MA regimens as inductive chemotherapy and then took autologous stem cell transplantation (ASCT) as consolidation treatment. Intrathecal chemotherapy was done in patients with $\mathrm{CNS}$ involvement. Eleven patients (27.5\%) had reached partial remission (PR), and fifteen patients (37.5\%) with complete remission or uncertain complete remission $(\mathrm{CR} / \mathrm{CRu})$. Therefore, the overall response rate (ORR) was $62.5 \%$. The ORR and CR/CRu for the 17 patients treated with rituximab was $76.4 \%$ and $52.9 \%$ respectively.

With a median follow-up of 18 months (range 2-93 months), 32 patients had died and the median follow-up for the surviving patients was 34.5 months (range 22-69 months). The median PFS and OS was 11.0 (95\% CI, 6.0-16.0) and 18.0 (95\% CI, 8.7-27.3) months, respectively. The median PFS and OS of the patients with CNS involvement were 5.0 (95\%CI, 4.1-5.9) and 8.0 (0.7-15.3) months, respectively, which were significantly shorter than those without CNS involvement (median PFS, 25.0 [range, 11.1-38.9 months], $p<0.001$; OS, 29.0 [range, 24.5-33.5 months], $p=0.01)$. DLBCL patients with concomitant BCL2 and $M Y C$ abnormalities also had a shorter median PFS (5.0 vs.22.5, $p=.002)$ and OS (9.0 vs.27.5, $p=.001$ ) than those without the genetic abnormality. Two of the patients receiving ASCT had disease progression after three months of ASCT, with one patient surviving over five years.

\section{Discussion}

Peripheral blood involvement in patients with DLBCL is rare. Currently, there are only three large studies have reported the frequency of $\mathrm{PB}$ involvement in DLBCL patients. In a Japanese study, $1.2 \%$ of patients with DLBCL demonstrated PB involvement [2], while two other studies from Western countries showed that $4 \%-5.3 \%$ of patients with DLBCL exhibited PB involvement [11, 12]. However, morphologic examination of peripheral blood smears revealed that approximately one third of DLB CL patients with BM involvement also had malignant cell in
PB. These studies did not assess the association between clinical and cytogenetic characteristics and $\mathrm{PB}$ involvement in DLBCL patients. To our knowledge, this study is the largest to report the clinical characteristics and outcome of DLBCL patients at leukemic phase $[6,13]$.

Similar clinical characteristics of leukemic DLBCL were identified in the study by Murungampurath-John [6], including the median age, gender, B symptom, bone marrow involvement, median $\mathrm{WBC}$ and $\mathrm{HB}$, and the distribution of IPI index. However, in our study, the number of extranodal site involvement was lower than that of a study by Murangampurath-John (50 vs. 100\%) [6]. CNS involvement was reported to be $22 \%$ in the study by Muringampurath-John [13], which is lower than that reported in our study (35\%). However, the reported number of CNS involvement in our study and the study by Muringampurath-John was higher than that reported in other DLBCL patient populations. When comparing the clinical characteristics between patients with or without CNS involvement, age was associated with CNS involvement. Additionally, when compared to the study by Muringampurath-John, the median WBC and PLT, and the percentage of circulating lymphomatous cells in this study were comparable, except for a higher percentage of patients with high IPI scores $(\geq 4)$ in that study (41 vs. 27.5\%) [6].

Cytogenetic aberrations are biologic and diagnostic hallmarks of mature B-cell lymphomas. The $\mathrm{t}(14 ; 18)$ (q32; $\mathrm{q} 21)$ translocation is the most common translocation observed in follicular lymphomas [1], which could potentially transform to DLBCL, [14]. In this study, 35\% DLBCL patients had BCL2 gene gain/amplification and none were reported having the $\mathrm{t}(14 ; 18)$ translocation, which was consistent with no patients having FL history. Recently, it was demonstrated that a double-hit lymphoma caused by multiple genetic aberrations, such as the MYC/8q24 locus and BCL2/18q21.3 locus can give rise to a unique subset of lymphomas. Translocation or amplification of the BCL2 gene occurred in $20-30 \%$ of cases of reported lymphomas [15]. MYC rearrangement have been reported in up to $10 \%$ of an unselected series of cases and is associated with a complex pattern of genetic alterations [1]. Most of the $M Y C$ translocations occur with IG genes [16]. However, these cytogenetic aberrations have not been specially detected in DLBCL patients at leukemic phase. Moreover, gene rearrangement was the main aberration of BCL2/MYC in DLBCL other than gene gain/amplification. Some studies have also reported that patients with 18q21.3/BCL2 and 8q24/MYC genetic rearrangement are at higher risk of having $\mathrm{CNS}$ involvement [16-18]. The incidence of CNS involvement ranged from $9 \%$ to $50 \%$ in double-hit (DH) lymphoma $[7,19,20]$. In this study, the incidence of CNS involvement increased to $88.9 \%$ in DLBCL patients at leukemic 
phase with concomitant $B C L 2$ and $M Y C$ gain/amplification. However, for patients with primary lymphoma of the CNS (PCNSL), it was reported that up to $8 \%$ had $M Y C$ rearrangement and none with $B C L 2$ rearrangements [21]. Therefore, the underlying mechanism of CNS involvement of leukemic DLBCL may differ from PCNSL.

PB or CNS involvement or concomitant genetic abnormalities of $M Y C$ and $B C L 2$ have been reported to be associated with poor survival in DLBCL $[2,16,18]$. Patients with leukemic phase have lower CR rate (44\%) even after rituximab combination chemotherapy, indicating drugresistance for this population [13]. In this study, the CR/ $\mathrm{CRu}$ rate for the patients treated with rituximab was $52.9 \%$, comparable to previous report (54\%) [6]. In this study, the median OS for all patients was 18 months, with CNS involvement and both BCL2/MYC dual abnormalities being predictors of poor clinical in DLBCL patients of leukemic phase. Three patients had received ASCT in this study. Two patients with CNS involvement reached $\mathrm{CR} / \mathrm{CRu}$ after R-HyperCVAD/MA introductive chemotherapy but had disease progression even after ASCT. The other patient remains alive after ASTC, and did not have CNS involvement. This phenomenon indicates that new chemotherapy or targeted therapy is needed for these patients.

\section{Conclusions}

Our study demonstrated that patients with DLBCL of leukemic phase had higher incidence of CNS involvement and concomitant BCL2 and MYC gene gains/amplifications. The concomitant of BCL2 and MYC gene gains/amplifications was the only independent factor that correlated with CNS involvement. Additionally, these patients exhibited a poorer treatment response and survival despite combination therapy with rituximab and ASCT.

\section{Abbreviations}

ASCT: Autologous stem cell transplantation; CHOP: Cyclophosphamide, doxorubicin, vincristine, prednisone; CNS: Central nervous system; DLBCL: Diffuse large B-cell lymphoma; FISH: Fluorescence in situ hybridization; Hb: Hemoglobin concentration; IPI: International prognostic index; LDH: Lactate dehydrogenase; NHL: Non-Hodgkin lymphoma; OS: Overall survival; PB: Peripheral blood; PFS: Progression-free survival; PLT: Platelet number; R: Rituximab; WBC: White blood cell

\section{Acknowledgements}

None.

\section{Funding}

This work is supported by grants from National Nature Science Foundation of China (81200395, 81370632 and 81400092), the National Science and Technology supporting Program (2014BAI09B12), Fundamental Application and advanced technology research program of Tianjin (15JCYBJC25100, 15JCYBJC45500 and 15JCYBJC27900), National Public Health Grand Research Foundation (201202017), and National Public Health Grand Research Foundation (201202017)

\section{Availability of data and materials}

The underlying data in this manuscript cannot be released to the public source due to relevant local data protection laws, unless we get the permit from the ethic committee upon reasonable request. We had the permission from our hospital to access and use the flow cytometry database.

\section{Author's contributions}

DZ and SY analyzed the clinical data and wrote the manuscript; SY, RC, SZ and $\mathrm{CL}$ performed FISH; WL, ZL, RL, GA, ZY and YX collected the clinical records; KR, HW and LQ made the diagnosis; LQ designed the study and revised the manuscript. All authors read and approved the final manuscript.

\section{Conflict of Interest}

The authors have declared no conflicts of interest.

\section{Consent for publication}

Not applicable.

\section{Ethics approval and consent to participate}

The included study had been approved by the Ethics Committee of Blood Disease Hospital, Chinese Academy of Medical Sciences (NI2016001-EC-1). The participants who enrolled in the study provided written consent to participate.

\section{Author details}

${ }^{1}$ State Key Laboratory of Experimental Hematology, Institute of Hematology and Blood Disease Hospital, Chinese Academy of Medical Sciences and Peking Union Medical College, No.288, Nanjing road, Heping district, Tianjin 300020, China. ${ }^{2}$ Department of Hematology, Tianjin First Center Hospital, Tianjin, China.

Received: 6 June 2016 Accepted: 10 February 2017

Published online: 16 February 2017

\section{References}

1. Swerdlow SH, Campo E, Harris NL, Jaffe ES, Pileri SA, Stain H, Thiele J, Vardiman JW e. WHO Classification of Tumours of Haematopoietic and Lymphoid Tissues, 2008. Lyon: IARC; 2008.

2. Takahashi H, Tomita N, Yokoyama M, Tsunoda S, Yano T, Murayama K, Hashimoto C, Tamura K, Sato K, Ishigatsubo Y. Prognostic impact of extranodal involvement in diffuse large B-cell lymphoma in the rituximab era. Cancer. 2012;118(17):4166-72.

3. Villa D, Connors JM, Shenkier TN, Gascoyne RD, Sehn LH, Savage KJ. Incidence and risk factors for central nervous system relapse in patients with diffuse large B-cell lymphoma: the impact of the addition of rituximab to CHOP chemotherapy. Ann Oncol. 2010;21(5):1046-52.

4. Kumar A, Vanderplas A, LaCasce AS, Rodriquez MA, Crosby AL, Lepisto E, Czuczman MS, Nademanee A, Niland J, Gordon LI, et al. Lack of benefit of central nervous system prophylaxis for diffuse large B-cell lymphoma in the rituximab era: findings from a large national database. Cancer. 2012:118(11):2944-51.

5. Tomita N, Yokoyama M, Yamamoto W, Watanabe R, Shimazu Y, Masaki Y, Tsunoda S, Hashimoto C, Murayama K, Yano T, et al. Central nervous system event in patients with diffuse large B-cell lymphoma in the rituximab era. Cancer Sci. 2012;103(2):245-51.

6. Muringampurath-John D, Jaye DL, Flowers CR, Saxe D, Chen Z, Lechowicz MJ, Weisenburger DD, Bast M, Arellano ML, Bernal-Mizrachi L, et al. Characteristics and outcomes of diffuse large B-cell lymphoma presenting in leukaemic phase. Br J Haematol. 2012;158(5):608-14.

7. Aukema SM, Siebert R, Schuuring E, van Imhoff GW, Kluin-Nelemans HC, Boerma EJ, Kluin PM. Double-hit B-cell lymphomas. Blood. 2011:117(8):2319-31.

8. Xu W, Li JY, Pan JL, Qiu HR, Shen YF, Li L, Wu YF, Xue YQ. Interphase fluorescence in situ hybridization detection of cytogenetic abnormalities in B-cell chronic lymphocytic leukemia. Int J Hematol. 2007;85(5):430-6.

9. $X u$ W, Li JY, Wu YJ, Yu H, Shen QD, Li L, Fan L, Qiu HX. Prognostic significance of ATM and TP53 deletions in Chinese patients with chronic lymphocytic leukemia. Leuk Res. 2008;32(7):1071-7.

10. Mossafa H, Damotte D, Jenabian A, Delarue R, Vincenneau A, Amouroux I, Jeandel R, Khoury E, Martelli JM, Samson T, et al. Non-Hodgkin's lymphomas 
with Burkitt-like cells are associated with c-Myc amplification and poor prognosis. Leuk Lymphoma. 2006;47(9):1885-93.

11. Arber DA, George TI. Bone marrow biopsy involvement by non-Hodgkin's lymphoma: frequency of lymphoma types, patterns, blood involvement, and discordance with other sites in 450 specimens. Am J Surg Pathol. 2005;29(12):1549-57.

12. Sovani V, Harvey C, Haynes AP, McMillan AK, Clark DM, O'Connor SR. Bone marrow trephine biopsy involvement by lymphoma: review of histopathological features in 511 specimens and correlation with diagnostic biopsy, aspirate and peripheral blood findings. J Clin Pathol. 2014;67(5):389-95.

13. Muringampurath-John $D$, Flowers $C R$, Jabbar $A A$, Sinha $R$, Arellano $M$, Bernal-Mizrachi L, Kaufman JL, Jaye DL, Chen Z, Lechowicz MJ, et al. Prognostic Significance of Circulating Lymphoma Cells in Newly Diagnosed Diffuse Large B-Cell Lymphoma (DLBCL). In: ASH Annual Meeting Abstracts, 22. 2009. p. 2947.

14. Barrans SL, Evans PA, O'Connor SJ, Kendall SJ, Owen RG, Haynes AP, Morgan GJ, Jack AS. The $t(14 ; 18)$ is associated with germinal centerderived diffuse large B-cell lymphoma and is a strong predictor of outcome. Clin Cancer Res. 2003;9(6):2133-9.

15. Iqbal J, Neppalli VT, Wright G, Dave BJ, Horsman DE, Rosenwald A, Lynch J, Hans $C P$, Weisenburger DD, Greiner TC, et al. BCL2 expression is a prognostic marker for the activated B-cell-like type of diffuse large B-cell lymphoma. J Clin Oncol. 2006;24(6):961-8.

16. Tomita N, Tokunaka M, Nakamura N, Takeuchi K, Koike J, Motomura S, Miyamoto K, Kikuchi A, Hyo R, Yakushijin Y, et al. Clinicopathological features of lymphoma/leukemia patients carrying both BCL2 and MYC translocations. Haematologica. 2009;94(7):935-43.

17. Le Gouill S, Talmant P, Touzeau C, Moreau A, Garand R, Juge-Morineau N, Gaillard F, Gastinne T, Milpied N, Moreau P, et al. The clinical presentation and prognosis of diffuse large B-cell lymphoma with $\mathrm{t}(14 ; 18)$ and 8q24/c-MYC rearrangement. Haematologica. 2007;92(10):1335-42.

18. Niitsu N, Okamoto M, Miura I, Hirano M. Clinical features and prognosis of de novo diffuse large B-cell lymphoma with $\mathrm{t}(14 ; 18)$ and $8 \mathrm{q} 24 / \mathrm{c}-\mathrm{MYC}$ translocations. Leukemia. 2009;23(4):777-83.

19. Petrich AM, Gandhi M, Jovanovic B, Castillo JJ, Rajguru S, Yang DT, Shah KA, Whyman JD, Lansigan F, Hernandez-llizaliturri FJ, et al. Impact of induction regimen and stem cell transplantation on outcomes in double-hit lymphoma: a multicenter retrospective analysis. Blood. 2014;124(15):2354-61.

20. Oki Y, Noorani M, Lin P, Davis RE, Neelapu SS, Ma L, Ahmed M, Rodriguez MA, Hagemeister FB, Fowler N, et al. Double hit lymphoma: the MD Anderson Cancer Center clinical experience. Br J Haematol. 2014;166(6):891-901.

21. Brunn A, Nagel I, Montesinos-Rongen M, Klapper W, Vater I, Paulus W, Hans V, Blumcke I, Weis J, Siebert R, et al. Frequent triple-hit expression of MYC, BCL2, and BCL6 in primary lymphoma of the central nervous system and absence of a favorable MYC(low)BCL2 (low) subgroup may underlie the inferior prognosis as compared to systemic diffuse large B cell lymphomas. Acta Neuropathol. 2013;126(4):603-5.

\section{Submit your next manuscript to BioMed Central and we will help you at every step:}

- We accept pre-submission inquiries

- Our selector tool helps you to find the most relevant journal

- We provide round the clock customer support

- Convenient online submission

- Thorough peer review

- Inclusion in PubMed and all major indexing services

- Maximum visibility for your research

Submit your manuscript at www.biomedcentral.com/submit

) Biomed Central 